\title{
FACTORES ASOCIADOS AL CONSUMO URBANO DE AGUA EN MÉXICO: LA IMPORTANCIA DE LA TARIFA
}

FACTORS ASSOCIATED WITH URBAN WATER CONSUMPTION IN MEXICO: THE IMPORTANCE OF RATE

\section{Hugo Briseño Ramírez}

hugo.briseno@up.edu.mx

\section{Jonathan Decle Castro²}

jonathandecle@hotmail.est

Fecha de recepción: Junio 28, 2016

Fecha de aceptación: Septiembre 22, 2016

\section{Forma sugerida de citar:}

\author{
Briseño Ramírez, Hugo [et.al.]< (2016). Factores \\ asociados al consumo Urbano de agua en \\ México: La importancia de la tarifa. Revista \\ Economía y Política. Año XII, No. 23. pp. 11-23
}

\section{Resumen}

En el siguiente trabajo se analizan los factores asociados al consumo urbano de agua en México. Se propone un modelo econométrico donde las variables explicativas del consumo son la tarifa de agua, el ingreso y la población por toma. Todas resultan significativas como señala la teoría.

\section{Palabras Clave}

consumo de agua en México, tarifa de agua, elasticidad precio.

\section{Abstract}

In the next document factors associated to water consumption in Mexico are analyzed. A model is proposed where explanatory variables of water consumption are water tariff, income and population by connection. All are significant as the theory suggest.

Keywords: water consumption, water tariffs, price elasticity.

\footnotetext{
1.Profesor de asignatura de la Universidad Panamericana campus Guadalajara-México. PhD en Ciencias Económico Administrativas. Universidad de Guadalajara.

2.Profesor de asignatura de la Universidad Juárez Autónoma de Tabasco. Maestría en Dirección y

Finanzas. Universidad Popular Autónoma del Estado de Puebla.
} 


\section{Introducción}

El crecimiento económico mundial requiere necesariamente el uso de recursos naturales. Estos recursos no son siempre renovables, por lo que es necesario un uso sustentable de los mismos. Este es el caso del agua potable, que es imprescindible para conservar la vida humana. Por lo tanto, debemos cuidarla y evitar a toda costa desperdiciarla; hacer todo lo posible para que la recarga de las fuentes de abastecimiento sea mayor a su extracción y no a la inversa. Es importante conocer los aspectos que influyen en el consumo del vital líquido.

Según la CONAGUA (2015), en el mundo solamente el $0.77 \%$ del agua es accesible para el ser humano; México tiene 2,467 municipios y delegaciones; el norte, noroeste y centro de México generan el $79 \%$ del PIB, concentran el $77 \%$ de la población y solo cuentan con el $33 \%$ del agua renovable; el sur y sureste de México generan el $21 \%$ del PIB, concentran el $23 \%$ de la población y cuentan con el $67 \%$ del agua renovable; de cada 100 litros de agua en México 77 son utilizados en uso agropecuario, 14 en abastecimiento público (9 de agua superficial y 5 subterránea), 4 en uso industrial y 5 en energía eléctrica; México tiene al menos 5,000 presas y bordos, de los cuáles 181 son grandes presas que concentran el $80 \%$ del almacenamiento total; en México el $66 \%$ del agua en los hogares se usa en baños y regaderas; y las tarifas por metro cúbico para un consumo de 30 metros cúbicos pueden variar de menos de 4 pesos a más de 20 pesos.
El objetivo de este trabajo es conocer los factores que determinan la demanda de agua para uso urbano en México. Para esto se formará un modelo con variables que hipotéticamente serán determinantes del consumo y se utilizará la herramienta estadística de la regresión para conocer si estas variables afectan, o no, el consumo y en qué grado. Las variables que resulten significativas serán consideradas como explicativas de la demanda.

En las siguientes páginas se explicará cómo se procedió a elaborar el modelo. Iniciaremos mencionando brevemente la teoría económica en donde se fundan nuestras hipótesis; en la segunda parte describiremos cómo se procedió a elaborar la base de datos y qué variables están incluidas en ésta, además de hacer un análisis estadístico; en la tercera parte se dará a conocer el modelo seleccionado y sus diferentes pruebas; y para terminar procederemos a interpretar los resultados y a ofrecer una breve conclusión sobre lo tratado en todo el documento.

La utilidad de este trabajo será conocer si las variables propuestas como explicativas de la demanda realmente influyen en ésta y en qué grado. Por otro lado, la función de la demanda servirá para hacer estudios de prospección sobre los diferentes municipios de México. 


\section{Teoría económica}

El presente trabajo se sustenta en la teoría de demanda que surge de un proceso de maximización de la utilidad individual sujeta a una restricción presupuestaria, enfoque conocido como Marginalista (Viscencio, 2002). Así, en términos generales, es posible establecer un modelo de demanda como:

$$
Q d_{x}=f\left(P_{x}, Y, P_{r}, Z\right)
$$

Donde $Q d_{x}$ representa la cantidad demandada de un bien o servicio, que está en función del precio propio del bien $\left(P_{x}\right)$, del ingreso del consumidor $(Y)$, del precio de bienes relacionados $\left(P_{r}\right)$, y de otros determinantes $(Z)$. Con las siguientes relaciones teóricas:

$\frac{\partial Q d_{x}}{\partial P_{x}}<0$, que indica que un cambio en el precio del bien, ceteris páribus, provoca una variación en la demanda en sentido opuesto. De esta forma, una subida en el precio genera disminución en la demanda. Este principio teórico es conocido como la "Ley de la Demanda" (Nicholson, 1997).

Por su parte, la teoría prevé que la relación ingreso - demanda es ambigua, dependiendo el tipo de bien. Si el bien es normal, un aumento en el ingreso se asocia con un incremento en la demanda del bien $\left(\frac{\partial Q d_{x}}{\partial Y}>0\right.$; pero si el bien es inferior, la relación ingreso - demanda es inversamente proporcional $\left(\frac{\partial Q d_{x}}{\partial Y}<0\right)^{3}$.

En cuanto a la relación entre demanda de un bien y el precio de otros bienes asociados, la teoría advierte también un efecto ambiguo. Si la relación es directa $\left(\frac{\partial Q d_{x}}{\partial P_{r}}>0\right)$, se trata de bienes sustitutos, y si la relación es inversa $\left(\frac{\partial Q d_{x}}{\partial P_{r}}<0\right)$, de bienes complementarios.

Finalmente, la demanda de un bien específico puede afectarse por diversas variables que responden a su naturaleza $(Z)$, pudiendo ser éstas de orden geográfico, social, etc., como por ejemplo el tamaño de la población, clima, temperatura, altura, etc. (Viscencio, 2002).

Con este sustento el modelo de consumo de agua quedaría determinado de la siguiente manera:

$$
C=f(T, Y, P O T)
$$

Donde $C$ representa el consumo de agua, $T$ la tarifa, $Y$ el ingreso y POT la población por toma. Para este modelo no se tomarán en cuenta los bienes asociados debido a la dificultad de encontrar información confiable, y a la ambigua relación que podría existir con el recurso hídrico.

3. Este hecho se refiere al principio de Engels según el cual una relación directa ingreso - consumo se asocia a bienes de tipo normal, como el caso de productos duraderos; y una relación inversa tiene que ver con bienes inferiores cuyo consumo disminuye ante un aumento del ingreso como el caso de la transportación pública, por ejemplo (Varian, 1993). 
Asumiendo una función exponencial (Cobb-Douglas) es posible especificar (2) de la siguiente manera:

$$
c=\varphi(T)^{\alpha}(Y)^{\beta}(P O T)^{\delta}
$$

Con $\varphi, \alpha, \beta, \delta$ coeficientes; $T$ referida a la tarifa promedio de agua por metro cúbico; $Y$ al ingreso promedio de la población; y POT a la población por toma.

Haciendo lineal la ecuación, mediante la aplicación de logaritmos, (3) quedaría de la forma:

$$
\ln (C)=\ln (\varphi)+\alpha \ln (T)+\beta \ln (Y)+\delta \ln (\mathrm{POT})
$$

A partir de (4) es posible determinar el modelo econométrico a ser estimado. Así tenemos:

$$
\ln (C)=\beta_{0}+\beta_{1} \ln (T)+\beta_{2} \ln (Y)+\beta_{3} \ln (\mathrm{POT})+\varepsilon
$$

Donde, a diferencia de (4), los coeficientes se representan por $\beta_{i}$ y se incluye el término de error $\varepsilon$.

Tras el fundamento teórico se espera que $\beta_{1}<0$, dando cumplimiento a la ley de la demanda; $\beta_{2}>0$ bajo el supuesto de que el bien agua es normal; y $\beta_{3}<0$ dado que dicho consumo disminuye con la población por toma por economías de escala.

Cabe señalar que, por los datos disponibles, se está considerando un modelo uniecuacional. Por lo que existe un riesgo de simultaneidad entre la variable explicativa y dependiente (sobre todo en precio y consumo), lo que puede generar tanto sesgo como inconsistencia en mínimos cuadrados ordinarios (Gujarati \& Porter, 2010; Wooldridge, 2015). Existen métodos para solucionar estos problemas, sin embargo requieren información adicional a la recabada y no es el propósito fundamental de este documento. Siendo esencial hacer resaltar la importancia de una tarifa adecuada que permita cobrar lo que cuesta llevar agua a la población.

\section{Base de datos y su análisis}

\section{Variables}

Tal como se sustenta en la teoría económica, se considera que la demanda de agua está en función de la tarifa de agua, el ingreso y la población por toma (por economías de escala). 
Dados estos supuestos, la función de demanda de agua quedaría de la siguiente manera:

$C=f(-T, Y,-P O T)$

Donde:

$$
\begin{aligned}
& C=\text { Cantidad demandada de agua } \\
& T=\text { Tarifa del agua } \\
& Y=\text { Ingreso } \\
& P O T=\text { Población por toma }
\end{aligned}
$$

En la tabla 1 podemos observar un resumen en el que se muestran las variables que se

\begin{tabular}{|c|c|c|}
\hline Variable & Fuente & Cálculo \\
\hline $\begin{array}{l}\text { Consumo de } \\
\text { Agua }(\mathrm{C})\end{array}$ & $\begin{array}{l}\text { Situación del Subsector } \\
\text { Agua Potable, Drenaje y } \\
\text { Saneamiento. Edición } 2014 \\
\text { CONAGUA. }\end{array}$ & $\begin{array}{l}\text { Litros por Habitante al Día } X \\
\text { (1 -Agua no contabilizada) }= \\
\text { Litros por Habitante al Día Reales o } \\
\text { Libres de Fugas }(C)\end{array}$ \\
\hline $\begin{array}{l}\text { Tarifa Promedio } \\
\text { de Agua }(\mathrm{T})\end{array}$ & $\begin{array}{l}\text { Situación del Subsector } \\
\text { Agua Potable, Drenaje y } \\
\text { Saneamiento. Edición } 2014 \\
\text { CONAGUA. }\end{array}$ & $\begin{array}{l}\text { Monto Facturado en Pesos / } \\
\text { Monto Facturado en } \mathrm{m}^{3}= \\
\text { Tarifa Promedio }(\mathrm{T})\end{array}$ \\
\hline Ingreso $(\mathrm{Y})$ & $\begin{array}{l}\text { Instituto Nacional de } \\
\text { Estadística y Geografía } \\
\text { (INEGI ) en IMCO (2014) }\end{array}$ & $\begin{array}{l}\text { Ingreso laboral promedio en pesos } \\
\text { mexicanos (por Estado) }\end{array}$ \\
\hline $\begin{array}{l}\text { Población por } \\
\text { Toma } \\
\text { (POT) }\end{array}$ & $\begin{array}{l}\text { Situación del Subsector } \\
\text { Agua Potable, Drenaje y } \\
\text { Saneamiento. Edición } 2014 \\
\text { CONAGUA. }\end{array}$ & $\begin{array}{l}\text { Población Atendida / } \\
\text { Número de Tomas = } \\
\text { Población por Toma (POT) }\end{array}$ \\
\hline
\end{tabular}
utilizaron para realizar el modelo, la fuente de información donde se obtuvieron y la forma de calcular los valores.

Tabla 1. Descripción de Variables

Fuente: elaboración propia con información de CONAGUA (2014) e IMCO (2014), referentes a los años 2013 y 2012 respectivamente.

La variable consumo de agua (C) son los litros consumidos por habitante al día reales. Es decir, los litros por habitante al día sustrayendo el efecto del agua no contabilizada. 
El agua no contabilizada es el agua que es suministrada a las redes de distribución de agua pero que nunca es facturada, ya sea porque se tira al subsuelo $o$ porque no se mide. Es importante restar al consumo por habitante el efecto del agua no contabilizada para poder medir correctamente el consumo que es facturado y que es influenciado por la tarifa y por el ingreso de los individuos.

Para obtener la tarifa de agua ( $T$ ) se dividieron los ingresos por servicios en pesos mexicanos entre el volumen vendido en $\mathrm{m} 3$. De esta manera se obtiene una tarifa promedio por metro cúbico.

Se espera que a mayor ingreso $(Y$ ) las personas consuman más agua debido a que realizan más actividades donde se utiliza este recurso. Ejemplos de estas actividades son: lavar más cantidad de ropa que la que lavarían personas de escasos recursos, lavar el vehículo, regar el jardín,..., por citar algunos.

La población por toma ( $P O T$ ) es el número de personas atendidas dividido entre el número de tomas que existen en un municipio. Este dato es importante porque entre más personas habiten en una residencia, se espera que su consumo per cápita sea menor ya que parte del uso que le darán al agua será común (economías de escala).

\section{Observaciones}

La base de datos cuenta con 80 observaciones referentes a ciudades con más de 50 mil habitantes en el año 2013. El criterio de selección de estos municipios fue que en el informe Situación del Subsector de Agua Potable, Drenaje y Saneamiento Edición 2014, publicado por la Comisión Nacional del Agua (CONAGUA), estuvieran disponibles sus datos de: litros por habitante al día, agua no contabilizada (o su inverso eficiencia física), ingresos por servicios, volumen vendido en metros cúbicos, población atendida y número de tomas. Era muy importante tener esta información, ya que sin ella no se podrían calcular variables esenciales para el modelo como son los litros por habitante al día reales, la tarifa promedio y la población por toma.

\section{Análisis estadístico}

En esta sección, examinaremos el comportamiento de las variables en la base de datos. En la tabla 2 podemos observar la estadística descriptiva para cada variable. Su media, su mediana y su desviación estándar; además de máximos y mínimos.

A continuación describiremos el comportamiento estadístico de cada variable:

Consumo. El promedio del consumo real en México (agua contabilizada o facturada) es de 174 litros por habitante al día. El consumo máximo es de 410 correspondiente a San Luis Río Colorado, Sonora; y el mínimo es de 46 correspondiente a Oaxaca de Juárez, Oaxaca. Existen datos extremos que están 
arrastrando ligeramente la media hacia valores grandes. Esto se comprueba al observar la mediana de 167 que es menor que la media de 174.

Tarifa promedio de agua. La media de la tarifa promedio de agua en México es de 10.96 pesos por metro cúbico vendido. La tarifa máxima es de 25.52 correspondiente a Tijuana, Baja California; y la mínima es de 0.84 correspondiente a Tepeapulco, Hidalgo.

Tabla 2. Estadística descriptiva de variables

\begin{tabular}{|c|c|c|c|c|c|}
\hline Variable & Media & Mediana & $\begin{array}{c}\text { Desviación } \\
\text { Estándar }\end{array}$ & Máximo & Mínimo \\
\hline $\mathrm{C}(\mathrm{l} / \mathrm{h} / \mathrm{d})$ & 174.57 & 167.16 & 65.95 & 410.05 & 46.53 \\
\hline $\mathrm{T}\left(\$ / \mathrm{m}^{3}\right.$ vendido) & 10.96 & 10.79 & 5.32 & 25.52 & 0.84 \\
\hline $\mathrm{Y}(\$$ ingreso promedio) & $\$ 5,518$ & $\$ 5,346$ & $\$ 645$ & $\$ 7,173$ & $\$ 3,444$ \\
\hline $\begin{array}{c}\text { POT (Población por } \\
\text { toma) }\end{array}$ & 3.45 & 3.29 & 0.65 & 5.98 & 2.55 \\
\hline ef (\%) & 54.73 & 54.51 & 14.11 & 85.71 & 30.18 \\
\hline
\end{tabular}

Fuente: elaboración propia con información de CONAGUA (2014) e IMCO (2014)

Nota: Se incluyó en esta tabla la eficiencia física (ef) por su influencia en el consumo de agua real.

Ingreso. La media del ingreso promedio en México (suponiendo que el dato estatal es igual al municipal) es de 5,518 pesos. El ingreso máximo es de 7,173 correspondiente al Distrito Federal y el mínimo es de 3,444 correspondiente al Estado de Chiapas.

Población por toma. El promedio de la población por toma de agua en México es de 3.45 habitantes. La población por toma máxima es de 5.98 correspondiente a Ciudad Netzahualcóyotl, México; y la mínima es de 2.55 correspondiente a Ciudad Obregón, Sonora.

Eficiencia física. El promedio de eficiencia física en México es de 54.73 por ciento; con un máximo de 85.71 correspondiente a Tecate, Baja California, y un mínimo de 30.18 correspondiente a Chetumal, Quintana Roo. 


\section{Modelo Econométrico}

En el presente apartado describiremos el proceso que se siguió para llegar al modelo de regresión seleccionado como el más apto para explicar la demanda de consumo de agua para uso urbano en México.

Tabla 3. Modelo econométrico (80 observaciones)

\begin{tabular}{|c|c|c|c|}
\hline $\begin{array}{c}\text { Variables } \\
\text { explicativas de InC }\end{array}$ & Coeficiente & Estadistico $t$ & Valor $\boldsymbol{p}$ \\
\hline Constante & 0.668619 & 0.2520 & 0.8018 \\
\hline $\operatorname{InT}$ & -0.214910 & -3.568 & $0.0006^{\star \star \star}$ \\
\hline $\ln \mathrm{O}$ & 0.734069 & 2.421 & $0.0178^{\star \star}$ \\
\hline $\operatorname{InPOT}$ & -1.15658 & -5.545 & $0.0000^{\star \star \star}$ \\
\hline
\end{tabular}

Fuente: elaboración propia con el soporte del programa GRETL

En la tabla 3 se muestra el modelo seleccionado como el mejor, entre los que se intentaron realizar, para explicar los determinantes del consumo de agua para uso urbano en México. Podemos observar que todas las variables son significativas al menos al 5 por ciento. El modelo también cumple satisfactoriamente con significancia conjunta, con normalidad de los errores, con homocedasticidad, y se encuentra correctamente especificado.

Tabla4. Pruebas estadísticas ${ }^{4}$ (normalidad, correcta especiación y homocedasticidad)

\begin{tabular}{|c|c|c|c|c|}
\hline Test & Hipótesis nula & Estadístico & $\begin{array}{c}\text { Valor } \\
\mathrm{p}\end{array}$ & $\begin{array}{c}\text { Hipótesis nula } \\
\text { aceptada o } \\
\text { rechazada }\end{array}$ \\
\hline Normalidad & $\begin{array}{c}\text { El error se distribuye } \\
\text { normalmente }\end{array}$ & 0.19 & 0.91 & Aceptada \\
\hline $\begin{array}{c}\text { Correcta especificación } \\
\text { (Reset) }\end{array}$ & $\begin{array}{c}\text { La especificación es } \\
\text { correcta }\end{array}$ & 0.58 & 0.56 & Aceptada \\
\hline Heterocedasticidad & $\begin{array}{c}\text { No hay } \\
\text { (White) }\end{array}$ & 8.95 & 0.44 & Aceptada \\
\hline
\end{tabular}

Fuente: elaboración propia con soporte del programa GRETL

4. Para profundizar en estas pruebas ver Gujarati y Porter (2010), y Greene (2003). 
El modelo resultante cumple con significancia conjunta como lo indica el estadístico $\mathrm{F}$ de 17.34 y el valor $\mathrm{p}$ de cero. La constante no resultó ser significativa. Las variables InT y InPOT son significativas al uno por ciento; y la variable InY es significativa al cinco por ciento. El coeficiente de determinación R2 es de 0.41 y el R2 corregido es 0.38 . En la tabla 4 podemos observar los resultados de las pruebas de normalidad, correcta especificación y homocedasticidad. El modelo satisface todas las pruebas antes mencionadas.

\section{Tabla 5. Matriz de correlación.}

\begin{tabular}{|c|c|c|c|c|}
\hline & $\operatorname{InC}$ & $\operatorname{InT}$ & $\operatorname{InY}$ & $\operatorname{InPOT}$ \\
\hline $\operatorname{InC}$ & 1 & & & \\
\hline $\operatorname{InT}$ & -0.2456 & 1 & & \\
\hline $\operatorname{In} Y$ & 0.2689 & 0.2029 & 1 & \\
\hline $\operatorname{InPOT}$ & -0.5318 & -0.0619 & -0.2202 & 1 \\
\hline
\end{tabular}

Fuente: elaboración propia con soporte del programa GRETL

Es importante evaluar si no hay presencia de multicolinealidad. Un criterio es observar si la correlación entre las variables explicativas ( $\ln T$, InY y InPOT) es menor a 0.5. En la tabla 5 podemos observar que ninguna de las correlaciones supera el 0.5 , por lo que podemos suponer, según el criterio empleado, que no hay multicolinealidad en el modelo.

Como se había señalado anteriormente, es importante reconocer que el modelo posiblemente cuente con endogeneidad. Esto debido a que la tarifa, en gran parte de México, tiene una estructura escalonada. Es decir, a mayor consumo va incrementando lo que se le cobra al usurario por metro cúbico. En otras palabras, decimos que puede existir simultaneidad porque la tarifa determina el consumo pero también el consumo determina la tarifa. Aunque no es objeto de este documento resolver este posible problema, es importante reconocer esta debilidad que pudiera estar presente.

\section{Interpretación de resultados}

En esta sección se interpretarán los resultados de acuerdo a las formas funcionales de las variables. Además, se generará una ecuación para estimar el consumo de agua de las localidades.

En la tabla 6 podemos observar las formas funcionales de las variables, sus coeficientes beta y la interpretación del impacto en el consumo de agua que es generado cuando hay cambios en las variables explicativas. 
Tabla 6. Interpretación de coeficientes (variables explicativas de InC)

\begin{tabular}{|c|c|c|l|}
\hline Variables X's & $\begin{array}{c}\text { Forma } \\
\text { funcional }\end{array}$ & $\begin{array}{c}\text { Coeficientes } \\
\beta \text { 's }\end{array}$ & \multicolumn{1}{|c|}{ Interpretación } \\
\hline $\operatorname{lnT}$ & $\log -\log$ & -0.214910 & $\begin{array}{l}\text { Un incremento de 1\% en la tarifa } \\
\text { disminuye en 0.21\% el consumo } \\
\text { de agua }\end{array}$ \\
\hline $\ln Y$ & $\log -\log$ & 0.734069 & $\begin{array}{l}\text { Un incremento de 1\% en el } \\
\text { ingreso aumenta en } 0.73 \% \text { el } \\
\text { consumo de agua }\end{array}$ \\
\hline $\operatorname{lnPOT}$ & $\log -\log$ & -1.15658 & $\begin{array}{l}\text { Un incremento de 1\% en la } \\
\text { población por toma disminuye } \\
\text { en 1.16\% el consumo de agua }\end{array}$ \\
\hline
\end{tabular}

Fuente: elaboración propia

Como muestra la tabla 6 , un incremento de $1 \%$ en la variable tarifa disminuye en $0.21 \%$ el consumo de agua; un incremento de $1 \%$ en el ingreso aumenta en $0.73 \%$ el consumo de agua; y un incremento de $1 \%$ en la población por toma reduce en $1.16 \%$ el consumo de agua.

Se puede intuir, que el agua para uso urbano tiene una demanda inelástica ante la tarifa. Sin embargo sí existe un impacto que es conveniente sea analizado por los tomadores de decisiones como una medida para reducir el consumo del líquido.

En cuanto al ingreso podemos concluir que el agua es un bien normal, ya que su consumo aumenta con un incremento en el ingreso. En este punto hay que señalar que además de tener una tarifa que cobre lo que realmente se consuma y castigue el desperdicio, es importante sensibilizar a la población para que ahorre tan necesario y escaso recurso.

La ecuación para estimar el consumo de agua se puede expresar de la siguiente manera:

$C=e^{\left[\left(-0.214910^{*} \ln T\right)+\left(0.734069^{*} \ln Y\right)-\left(1.15658^{*} \ln P O T\right]\right.}$

O de esta otra:

$C=(T-0.214910)(Y 0.734069)(P O T-1.15658)$ 


\section{Conclusiones}

Según los resultados de la regresión, la demanda de agua está determinada por la tarifa promedio, el ingreso laboral medio de los consumidores y la población por toma. Según este modelo, un incremento en la tarifa de agua en $1 \%$ tiene un impacto de $0.21 \%$ en la reducción de consumo de agua; un incremento de $1 \%$ del ingreso aumenta el consumo en $0.73 \%$; y un incremento del $1 \%$ en la población por toma reduce el consumo per cápita en $1.16 \%$ por el efecto de economías de escala.

Si se busca reducir el consumo de agua por habitante, una alternativa que tiene el gobierno es incrementar la tarifa. Incrementar la tarifa es algo políticamente incorrecto pero económicamente necesario en algunos municipios. Por un lado provoca que la gente valore más el servicio que los organismos operadores de agua prestan al llevar este recurso a los domicilios y, por lo tanto, modere su consumo; y por otro, incrementa la utilidad o disminuye la pérdida financiera de los organismos operadores, quienes necesitan recursos para sus procedimientos y para la reparación de las redes de distribución.

Existe un problema generalizado que consiste en que los organismos operadores tengan tarifas que no alcancen ni a cubrir los costos operativos. Esto se debe a que los organismos operadores no tienen la autonomía y la autoridad para solicitar que la tarifa se incremente; por el contrario, existen incentivos del sistema político de incrementar los costos contratando más personal (Ehrhardt \& Janson, 2010).

El incentivo político es muy importante a la hora de tomar decisiones sobre el incremento de la tarifa. Ésta no se determina bajo un razonamiento técnico. El incremento de la tarifa será diferente dependiendo de quién lo determine. Si la decisión es tomada por un decisor con criterios políticos, el incremento tenderá a ser insuficiente o nulo; si la decisión es tomada por un consejo consultivo independiente tenderá a ser técnica. En estos dos extremos existen varias opciones intermedias. Es interesante darle seguimiento al caso de Guadalajara donde ya existe una Comisión Tarifaria que busca lograr una tarifa sustentable, ajena a criterios políticos, para que el organismo encargado de suministrar el agua pueda cubrir los costos del servicio (Briseño, Flores, Pérez \& Salazar, 2016).

Es necesario que las autoridades encargadas de definir la tarifa, tomen consciencia de que es mayor daño el que se le hace a la población dejando a los organismos operadores de agua sin recursos financieros y permitiendo que la gente desperdicie el agua. Se conservan estas políticas en nombre de los pobres cuando son estos los menos beneficiados. La gente que no tiene acceso al agua necesita contratar pipas que le proporcionen el servicio y esto es más caro que pagar una tarifa razonable. "Por ejemplo, las viviendas sin conexión en las ciudades fronterizas del norte de México tienen que pagar hasta más de cinco dólares por un metro cúbico de agua; en 
contraste, las viviendas conectadas pagan medio dólar por la misma cantidad de agua" (Aguilar \& Saphores, 2009, 3).

Además, no necesariamente se les debe subir la tarifa a todos. Se puede diseñar un esquema en el que, los que menos tienen, paguen una tarifa más baja. Otro aspecto a considerar, es que pagamos indirectamente la tarifa del agua a través de otros impuestos, porque los organismos operadores deben ser "apoyados" por el gobierno para ser financieramente viables.

Si se toma como instrumento de política pública para reducir la demanda el incremento de la tarifa, es necesario que esto vaya acompañado de una penalización a quien no paga. Porque, si en la estado actual hay morosidad e incumplimiento de pago, esta situación se agravará con el incremento de la tarifa. De no hacer nada, el resultado sería el mismo, porque al consumidor le dará lo mismo pagar que no pagar; y ni la demanda disminuirá, ni la situación financiera del organismo mejorará.

Spiller \& Savedoff (1997) señalan que existe un equilibrio de bajo nivel en el que no hay incentivos para pagar por el servicio y a la vez no se exige una mejora en el mismo. Es decir, la baja calidad del servicio se empata con el beneficio que obtiene el usuario al no pagarlo.

Es importante que existan diseños institucionales innovadores que puedan proveer de incentivos a los usuarios del servicio (Easter \& McCann, 2010) para pagar el agua y ahorrarla. El factor tarifa sigue siendo determinante, ya que mientras ésta sea baja, difícilmente sensibilizará en el ahorro de agua. $Y$ mientras no se castigue el no pago; no existirán incentivos para saldar la deuda de los usuarios por el uso del agua.

En suma, es de vital importancia que las autoridades facultadas para determinar la tarifa de agua, tomen conciencia de que en sus manos está parte importante de la salud financiera de los organismos operadores, de la moderación en el consumo de tan importante recurso, y de la viabilidad futura de las ciudades. 


\section{Referencias}

Aguilar-Benítez, I. \& Saphores, J.D. (2009). Aspectos Institucionales y Políticas para Reforzar el Pago de los Servicios del Agua en Nuevo Laredo, Tamaulipas y Laredo Texas. Nuevo León. Gestión y Política Pública. Vol. XVIII, No. 2.

Briseño, H., Flores, R., Pérez, G. \& Salazar, A. (2016). La gestión del agua potable en la Zona Metropolitana de Guadalajara. En Fugas de agua y dinero. Factores político-institucionales que inciden ene le desempeño de los organismos operadores de agua potable en México, coordinado por A. Salazar, 237 - 268. México: El Colegio de Sonora.

Comisión Nacional del Agua (CONAGUA) (2015). Numeragua México 2015. México, D.F.: Secretaría de Medio Ambiente y de Recursos Naturales.

Comisión Nacional del Agua (CONAGUA) (2014). Situación del Subsector Agua Potable, Drenaje y Saneamiento, Edición 2014. México, D.F.: Secretaría de Medio Ambiente y de Recursos Naturales.

Greene, W. (2003). Econometric Analysis. Estados Unidos: Pearson.

Gujarati, D. \& Porter, D. (2010). Econometría. México: McGraw - Hill.

Easter, K.W. \& McCann, L.M.J. (2010). Nested institutions and the need to improve international water institutions. Water Policy, 12, 500 - 516. doi: 10.2166/ wp.2010.002

Ehrhardt, D. \& Janson, N. (2010). Can regulation improve the performance of government - controlled water utilities? Water Policy, 12, S. I, 23 - 40. doi: 10.2166/ wp.2010.112

Instituto Mexicano para la Competitividad (IMCO) (2014). Índice de competitividad estatal 2014. Las reformas y los estados. La responsabilidad de las entidades en el éxito de los cambios estructurales. México: IMCO.

Nicholson, W. (1997). Teoría Microeconómica: Principios Básicos y Aplicaciones. McGraw- Hill. Sexta edición.

Spiller, P.T. \& W. Savedoff. (1997). 'Commitment and governance in infrastructure sectors', in Willig, Uribe and Basañes (eds.), Can Privatization Deliver Infrastructure for Latin America?, Balitmore, Maryland: Johns Hopkins University Press.

Varian, H. (1993). Microeconomía Intermedia, un Enfoque Moderno. Antoni Boch, tercera edición.

Viscencio, H. (2002). Economía para la toma de decisiones. México: Internacional Thomson Editores.

Wooldridge, J. (2015). Introducción a la econometría. México: CENGAGE Learning. 
